\title{
Assessment of patient dose in medical processes by in-vivo dose measuring devices: A review
}

\author{
Nina Tuncel \\ Akdeniz University- Science Faculty -Department of Physics, Antalya-Turkey
}

\begin{abstract}
In-vivo dosimetry (IVD) in medicine especially in radiation therapy is a well-established and recommended procedure for the estimation of the dose delivered to a patient during the radiation treatment. It became even more important with the emerging use of new and more complex radiotherapy techniques such as intensity-modulated or image-guided radiation therapy. While IVD has been used in brachytherapy for decades and the initial motivation for performing was mainly to assess doses to organs at risk by direct measurements, it is now possible to calculate 3D for detection of deviations or errors. Invivo dosimeters can be divided into real-time and passive detectors that need some finite time following irradiation for their analysis. They require a calibration against a calibrated ionization chamber in a known radiation field. Most of these detectors have a response that is energy and/or dose rate dependent and consequently require adjustments of the response to account for changes in the actual radiation conditions compared to the calibration situation. Correction factors are therefore necessary to take. Today, the most common dosimeters for patients' dose verification through in-vivo measurements are semiconductor diodes, thermo-luminescent dosimeters, optically stimulated luminescence dosimeters, metal-oxidesemiconductor field-effect transistors and plastic scintillator detectors with small outer diameters.
\end{abstract}

\section{Introduction}

There are many steps in the chain of processes which determine the absorbed dose delivered to patients in medicine and each of these steps may introduce uncertainties. It is therefore important to check the absorbed dose which has been delivered in practice by putting dosimeters on the patient's skin or in body natural cavities, by placing devices away from patient for detecting the transit photons or emission photons from patients. The ionometric dosimetry by different systems would be applied. This method is called invivo dosimetry (IVD). By growing awareness of the need to monitor the dose delivered to patients the implementation of IVD in clinical practice of radiotherapy is widely considered. By the way, in nuclear medicine, diagnostic/interventional procedures at computed tomography, and fluoroscopy/ interventional radiology the in-vivo dosimetry method seem on the rise.

\section{Radiotherapy}

The IVD in medicine especially in radiation therapy is a well-established and recommended procedure [1-3] for the estimation of the dose delivered to a patient during the radiation treatment. It provides an independent check of the treatment procedure that aims at detection of possible errors in calculation, patient setup and data transfer. In-vivo dosimetry is recognized as part of the quality assurance programme in radiotherapy. It became even more important with the emerging use of new and more complex radiotherapy techniques such as intensity-modulated (IMRT) or image-guided radiation therapy [4]. While IVD has been used in brachytherapy (BT) for decades and the initial motivation for performing was mainly to assess doses to organs at risk (OAR) by direct measurements, because precise evaluation of OAR doses was difficult without $3 \mathrm{D}$ dose treatment planning. With the introduction of 3D image-guided BT, it is now possible to calculate $3 \mathrm{D}$ tumour and OAR doses but IVD is very relevant in $\mathrm{BT}$ as an independent method for detection of deviations or errors [5].

Comprehensive guidelines for the implementation of in-vivo dosimetry with diodes are published by ESTRO [6] and AAPM [7]. Generally, in-vivo dosimeters are available as passive detectors with delayed readout or as real-time detectors with immediate readout. Although real-time techniques have obvious advantages over passive dosimeters, these are supplied immediate judgement possibility regarding to process. Currently 3D image-based dose distributions provide the possibility to determine patient absorbed dose and dose-volume histograms (DVHs) in-vivo at the new innovative techniques in external radiotherapy [8]. The other in-vivo applications for predicting patient absorbed dose after Proton therapy is PET-CT imaging modality $[9,10]$.

\section{Nuclear Medicine}

The early in-vivo tracer studies by external gamma ray counting used in nuclear medicine and have been 
progressively shifted from static measurements to dynamic measurements which are performed over a period of time. The variation of the concentration of radioactivity in any site provides the fundamental relationship to be analyzed. The concentration to be measured may occur in a number of different sites, organs, dilution volumes, or combinations of such functional entities. For example, the prior studies on bone were done using ${ }^{47} \mathrm{Ca}$ and ${ }^{85} \mathrm{~S}$ radioactive tracers and applied radionuclide scanning to determine and outline the probable site of lesion involved bone [11]. The first applications of external gamma ray counting or in-vivo tracer studies in medicine were the measurement of the uptake of 131-I by thyroid gland at a specific time following the administration of the dose. And additional information could be obtained by measuring the rate of uptake rather by calculating time concentration curves than the final asymptotic value. Then, understanding kinetics of radionuclides is evaluated in the other cases like liver, kidney and bone structures [12]. Nowadays the patient-specific dosimetry for 131-I thyroid cancer therapy using 124-I Positron emission tomography (PET) images which adapted to 3-dimensional-internal dosimetry (3D-ID) software is used for obtaining spatial distributions of absorbed dose, isodose contours, dose-volume histograms (DVHs), and mean absorbed dose estimates [13]. And for the same iodinetherapy cases in-vivo dosimetry performed using patient-specific 3dimensional methods for SPECT [14]. The fulfilment of such favourable conditions in actual clinical cases can only be confirmed by in-vivo quantization of the absorbed dose achieved as a result of the administration of radioiodine.

\section{Radiodiagnostic}

The diagnosis with radiation examinations using $\mathrm{x}$-ray tubes to obtain images is based in this field. IVD concept does not used directly but the patient dose is the subject by direct or indirect measurements. The principal quantities for patient dosimetry in general radiography are the incident air kerma, the entrance surface air kerma and the air kerma-area product. For each patient, the incident air kerma can be determined by calculation from recorded exposure parameters and the measured tube output. The entrance surface air kerma provides direct assessment of patient dose and can be measured by TLDs $[15,16]$. Radiology is an important technique for the diagnosis of patients and in recent years there has been an increase in the use of radiology as part of the treatment of patients. In general, radiation dose levels in radiology are highest for procedures involving fluoroscopy and computed tomography. An international study into patient dose levels in fluoroscopy and digital imaging techniques has been initiated using dose area product meters. A computerized method of automatically monitoring tube and generator parameters to perform on-line quality assurance and patient dosimetry has been developed $[17,18]$. Today, with the introduction of digital radiology, it is possible to have automatic systems to collect and archive patient dose data individually, for all the imaging and interventional procedures, in addition to demographic, geometric, and other procedural parameters, as part of the DICOM header or through other DICOM services [19].

\section{IVD: Passive and Active Methods}

In-vivo dosimeters can be divided into real-time and passive detectors that need some finite time following irradiation for their analysis. Both types of dosimeter require a calibration generally obtained by comparing their response against a calibrated ionization chamber (IC) in a known radiation field. Most of these detectors have a response that is energy and/or dose rate dependent and consequently require adjustments of the response to account for changes in the actual radiation conditions compared to the calibration situation. Correction factors are therefore necessary to take, for instance, changes in field size, source-detector distance, temperature, pressure, and orientation, including the presence of a build-up cap, into account. The presence of a build-up cap is important for detectors used for entrance or exit IVD [4].

Today, the most common dosimeters for patients' dose verification through in-vivo measurements are semiconductor diodes, thermoluminescent dosimeters (TLDs) and optically stimulated luminescence dosimeters (OSLDs), or electronic portal imaging devices. There is also increased application of metaloxide-semiconductor field-effect transistors (MOSFET) for clinical radiotherapy dosimetry. Diodebased in-vivo dosimetry has been widely explored and reported in the literature [20-24]. Similar investigations have been reported with TLDs $[25,26]$ and with MOSFET dosimeters [27-29]. On the other hand OSLDs potential in medical dosimetry [30] was recognized due to their small size suitable for point measurements, high sensitivity, non-destructive readout and reanalysis and the use of relatively simple readers with a high degree of automation. Technical advances in the production of OSL materials and availability of the reading devices make them a very promising dosimetric tool for medical purposes. Current developments and specific applications of OSLDs in diagnostic radiology and radiation therapy are reconsidered [31-33].

TLDs and OSLDs were the most common choice of passive dosimeters for medical purposes. However, OSLDs have an advantage compared with TLDs [34], since no heating is required for the dose measurement, which makes the reading equipment cheaper, and easier to handle and maintain. These are still widely used in routine work for several reasons. Usually there are a larger number of dosimeters provided for in-vivo measurements which allows for simultaneous dose determination at several positions on the patient. No cable connection between dosimeters and the reader allows their usage on different treatment machines in the department. Moreover, it is not necessary for the 
hospital to be equipped with the reading unit; instead, readout can be arranged in an outside institution.

The innovative solution of so-called in vivo "transit" dosimetry currently is being implemented in many radiotherapy centers. Usually used to check patient positioning, portal imagers (Electronic portal imaging device; EPID) allow two-dimensional acquisition of the signal transmitted through the patient throughout the entire duration of irradiation. It is then possible to arrive at an estimate of the dose in the patient. The dose thus reconstructed can then be compared to the planned dose to validate the quality of the treatment delivered. This method has a certain number of advantages compared to the "standard" method so measurement of the dose at a point by placing a detector on the skin and opens up a number of prospects with regard to strategies for validation of the dose delivered to the patient. Currently 3D EPIDbased dose distributions provide the possibility to determine patient absorbed dose and dose-volume histograms (DVHs) in-vivo at the new innovative techniques in radiotherapy [8]. The other applications for predicting patient absorbed dose after proton therapy is PET-CT imaging modality [10].The later methods that based on images and applied soft ware could be consider as real time in-vivo dosimetry.

\section{Conclusions}

IVD programs in radiotherapy contributed to increased expertise in radiation dosimetry in clinical environment leading to increased precision on treatment delivery, better detection of systematic error and the prevention accidents in radiotherapy. The innovative approach of so-called in-vivo "transit" dosimetry allows in vivo dosimetry to be extended to other fields of medicine. Studies are also underway on this approach.

\section{References}

1. AAPM Report No.46, Med. Phys.Med. Phys. 21, 581 (1994).

2. M. Essers, B.J. Mijnheer, Int. J. Radiat. Oncol. Biol. Phys. 43, 245 (1999).

3. IAEA, Setting Up a Radiotherapy Programme, , Vienna (2008).

4. B. Mijnheer, et al., Med. Phys. 40, 1 (2013).

5. K. Tanderup, et al., Med. Phys. 40, 1 (2013).

6. D. Huyskens, et al., (ESTRO Booklet No.5, Brussels, 2001).

7. AAPM Report No.87, AAPM (2005).

8. B. Mijnheer, et al., Journal of Physics: Conference Series 444, (2013).

9. K. Parodi, 68, 920-934 (2007).

10. X. Zhu, G. El Fakhri, 3, 731-740 (2013).

11. D. A. Weber, et al., J Nucl Med., 10, 8-17 (1969).

12. M. G. Stabin, Fundamental of Nuclear Medicine Dosimetry, (Springer, 2008).

13. G. Sgouros, et al., J. Nucl. Med., 45 (8) 1366 (2004).
14. Y. K. Dewaraja, et al., J. Nucl. Med., 46:840-849 (2005).

15. ICRU Report 74, (ICRU, Bethesda, MD, 2006).

16. IAEA, Technical Reports Series No. 457, (IAEA, Vienna, Austria, 2007).

17. K. Faulkner, et al., 12, 228-232 (1990).

18. A. Meghzifene, et al., European Journal of Radiology, 76, 11-14 (2010).

19. E. Vano, et al., AJR, 200, 783-790 (2013).

20. C. J. Millwater, A. S. MacLeod, D. I. Thwaites, Br. J. Radiol. 71, 661-8 (1998).

21. R. Alecu, T. Loomis, J. Alecu, T. Ochran, Med. Dosim. 24, 5-12 (1999).

22. N. Jornet, M. Ribas, T. Eudaldo, Med. Phys., 27, 1287-93 (2000).

23. C. Fiorino et. al, Radiother.Oncol., 56, 85-95 (2000).

24. P. A. Jursinic, Med. Phys., 28, 1718-26 (2001).

25. T. Loncol, et al., Radiother. Oncol., 41, 179 (1996).

26. A. Swinnen, J. Verstraete, D.P. Huyskens, Radiother. Oncol., 73, 89 (2004).

27. R. Ramaseshan, et al., M. Islam, Phys. Med. Biol., 49, 4031 (2004).

28. C.W. Scarantino, et al., Int. J. Radiat. Oncol. Biol. Phys., 62, 606-13(2005).

29. A. Cherpak, et al., Med.Phys., 36, 1672-9 (2009).

30. M.S. Akselrod, et al., Radiat. Meas., 41, 78 (2006).

31. E.G. Yukihara, S.W.S. McKeever, Phys.Med. Biol., 53, 351 (2008).

32. E. Yukihara, et al., Med. Phys., 35, 260 (2008).

33. E.G. Yukihara, et al., Radiat. Meas., 45, 658 (2010).

34. S.W.S. McKeever, M. Moscovitch, Radiat. Prot. Dosim., 104, 263-70 (2003). 